Journal Club

Editor's Note: These short, critical reviews of recent papers in the Journal, written exclusively by graduate students or postdoctoral fellows, are intended to summarize the important findings of the paper and provide additional insight and commentary. For more information on the format and purpose of the Journal Club, please see http://www.jneurosci.org/misc/ifa_features.shtml.

\title{
Characterizing the Involvement of Rostrolateral Prefrontal Cortex in Prospective Memory
}

\author{
Michael T. Rubens $\mathbf{s}^{1,2}$ and Theodore P. Zanto ${ }^{1,2}$ \\ Departments of ${ }^{1}$ Neurology and ${ }^{2}$ Physiology, University of California San Francisco, San Francisco, California 94158 \\ Review of Gilbert
}

To multitask, one must maintain an overarching objective while engaged in a subordinate task. The ability to hierarchically organize goals has been called cognitive branching, goal-subgoal integration, or prospective memory (PM). Functional neuroimaging has established that lateral aspects of Brodmann area 10 (BA10), referred to as the rostrolateral prefrontal cortex (RLPFC), facilitate this complex cognitive behavior (Burgess et al., 2001). However, the precise role of the RLPFC in hierarchically organizing behavior is unclear. Little empirical work has aimed at specifying which processing stage or stages (i.e., encoding, maintaining, or retrieving delayed intentions) depend upon the engagement of RLPFC regions during PM tasks, in which individuals must hold in mind a plan for a forthcoming action. Furthermore, it is unknown whether the RLPFC stores a "goal stack," representing the content of goals to be achieved, or whether it plays a content-independent role that subserves PM through functional connections with distinct, distal regions that store representations of one's intentions.

In a recent issue of The Journal of $\mathrm{Neu}$ roscience, Gilbert (2011) used functional

Received April 14, 2011; revised May 10, 2011; accepted May 13, 2011.

T.Z. was supported by a postdoctoral fellowship from the National Institutes of Health (4F32AG030249-03). We thank Drs. Silvia Bunge, Jesse Rissman, and Peter Wais for their comments and suggestions.

Correspondence should be addressed to Theodore Zanto, Departments of Neurology and Physiology, University of California San Francisco, 600 16th Street, Box 2240, San Francisco, CA 94158. E-mail: theodore.zanto@ucff.edu. DOI:10.1523/JNEUROSCI.1891-11.2011

Copyright $\odot 2011$ the authors $\quad 0270-6474 / 11 / 319067-03 \$ 15.00 / 0$ magnetic resonance imaging (fMRI) to establish which processing stage(s) engages the RLPFC, and whether the RLPFC stores the content of delayed intentions. Participants performed a two-back task, which involved pressing a button if the current stimulus matches the stimulus presented two items prior and responding with a different button if it did not match. Additionally, colored borders around randomly selected stimuli (pictures or words) cued participants to either encode the stimulus for subsequent recognition (PM multitasking trials) or continue the two-back task as usual (baseline trials). The fMRI data were interrogated through a comprehensive, multitiered analytic approach that incorporated univariate analysis, multivoxel pattern analysis (MVPA), and multivariate analysis of psychophysiological interactions (PPI).

To identify brain regions involved in each processing stage of the task, PM trials were contrasted against baseline trials in a whole-brain univariate analysis. Activity related to PM encoding, maintenance, and retrieval was observed in widespread cortical and subcortical regions, which were largely distinct between the task stages. Notably, univariate analyses indicate that bilateral RLPFC selectively becomes active during PM maintenance, and not during the encoding or retrieval stages of PM processes.

Subsequently, Gilbert (2011) used MVPA to assess whether the RLPFC is involved in storing the content of intentions during the maintenance period. The first MVPA established neural regions involved in decoding stimulus type (i.e., pictures or words). This analysis identified the medial rostral PFC, thalamus, right superior parietal cortex, and medial occipital cortex. Importantly, there was no evidence that the RLPFC was involved in storing the target of delayed intentions (i.e., stimulus content). The second MVPA identified regions related to the differential motor response stipulated by the PM task. Successful classification was observed in several regions, including motor cortex, putamen, medial temporal lobe, and lateral occipital cortex. Similar to the previous MVPA, this analysis suggests the RLPFC does not represent the nature of intentions (i.e., content of the motor response goals) during the maintenance period. However, to ensure these results were not due to a lack of power to detect an RLPFC effect, another MVPA was conducted to identify brain regions associated with classifying trial type (i.e., PM or baseline). This analysis revealed that bilateral RLPFC activity was related to whether delayed intentions were being maintained. Therefore, these results converge to suggest that widespread posterior regions are responsible for storing the content of intentions, whereas the RLPFC plays a non-content-specific role in facilitating delayed intentions.

Given the apparent content-independent role of the RLPFC in PM storage, Gilbert (2011) hypothesized that the RLPFC supports PM via long-distance communication with regions that store the procedural 


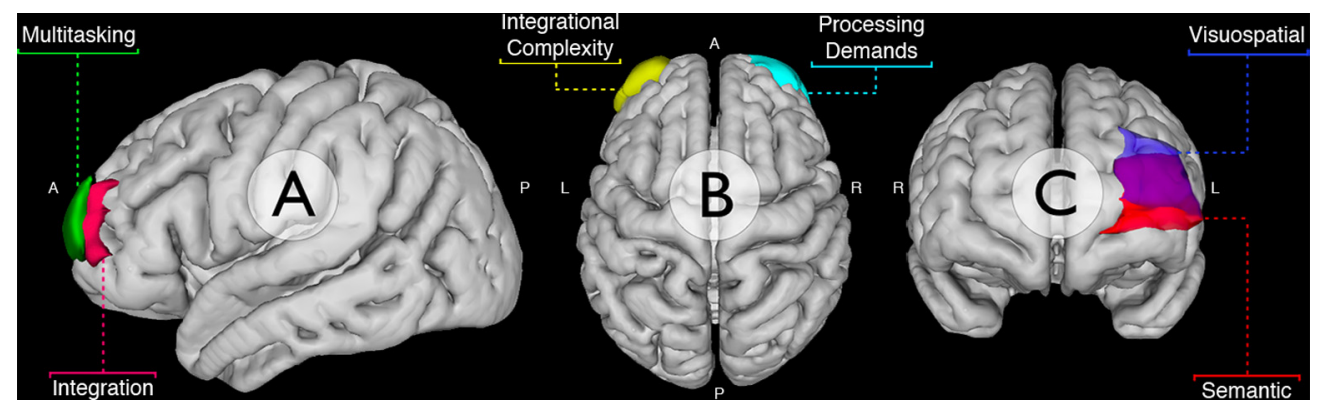

Figure 1. Illustration of prospective RLPFC functional organization. Previous research has suggested the RLPFC can be subdivided along the anterior-posterior axis $(\boldsymbol{A})$, by hemisphere ( $\boldsymbol{B})$, and along the dorsal-ventral axis (C). The areas characterized in this figure do not represent actual functional data.

information of intentions (i.e., content of task goals). To address this, a PPI analysis was used to determine which areas have a functional relationship with the RLPFC during the maintenance period. The RLPFC seed region was defined by taking all significant voxels within BA10 based on the univariate contrast of PM store $>$ baseline store. Results from the PPI analysis indicate that the RLPFC couples more strongly with posterior content-storing regions identified by MVPA, compared to areas identified by the univariate analysis. Thus, these results confirm the hypothesis that the RLPFC supports PM through functional interactions with distributed content-representing brain regions. Overall, Gilbert (2011) shows that intentions are likely represented in a distributed network of posterior brain regions, whereas the RLPFC directs the maintenance (as opposed to encoding or retrieval) of those representations to exercise a delayed intention at the appropriate time.

Gilbert (2011) suggests that future research should address the prospect that different aspects of delayed intentions may be represented within subregions of the RLPFC. Functional segregation of the RLPFC is plausible, because the PFC is thought to be organized hierarchically along the rostrocaudal axis, with more rostral portions supporting increasingly abstract neural representations and complex action rules (Badre and D'Esposito, 2009). Furthermore, the rostral portion of the PFC, BA10, may be further segregated in the medial-lateral dimension such that the RLPFC is sensitive to changes in demands for stimulus-independent attention as part of a supervisory attentional gateway (Burgess et al., 2007). The data from Gilbert (2011) supports these hypotheses by demonstrating that stimulusindependent complex action rules may be maintained by the RLPFC.

Accordingly, previous research has indicated that regions within the RLPFC may be functionally segregated. Studies of cognitive branching (Koechlin et al., 1999) and PM (Burgess et al., 2001) have elicited RLPFC activity in regions anterior to those activated during a relational reasoning task (Wendelken et al., 2008). This suggests that posterior areas of the RLPFC are related to integration processes (i.e., joint processing of multiple relations), whereas the anterior portion is involved in multitasking (Wendelken et al., 2008). This coincides with the general rostrocaudal organization of the PFC (Fig. 1A).

Although the reported coordinates of peak RLPFC activity during Gilbert's (2011) PM task are between activity elicited by relational reasoning (Wendelken et al., 2008) and a previous PM task (Burgess et al., 2001), the large cluster of activity reported by Gilbert (2011) appears to encompass both the anterior and posterior portions of the RLPFC. Thus, if the RLPFC is functionally segregated, this would suggest that both multitasking and integration processes were active during the PM task. This seems reasonable given that the experiment was designed to entail multitasking, yet required participants to integrate sensory cues with cognitive actions across time. Indeed, a primary function of the lateral PFC is the temporal integration of information to achieve prospective behavioral goals (Fuster, 2001).

Recent research into the role of the RLPFC has identified a left lateralization for integrational complexity, whereas the right RLPFC is more sensitive to processing demands (Fig. 1B) (Bunge et al., 2009). Furthermore, the right RLPFC has demonstrated functional connectivity with the hippocampus following relational encoding (Wendelken and Bunge, 2010). Interestingly, both Gilbert (2011) and Adda et al. (2008) also observed hippocampal activation during the PM encoding stage. Although the PPI analysis implemented by Gilbert (2011) did not identify RLPFC-hippocampal connectiv- ity, this may have been due to the fact that a bilateral RLPFC seed was used. Had a lateralized RLPFC seed been used, it is conceivable that the results would indicate that a portion of the RLPFC draws from hippocampal representations during the PM task via a functional connection. This analysis would not only help elucidate the role of the RLPFC during PM, but could also address potential laterality differences.

In addition to the potential for the RLPFC to be functionally segregated, there is accumulating evidence for a content-specific gradation along the dorsal-ventral axis within the RLPFC, such that tasks with visuospatial stimuli elicit left RLPFC activity that is more dorsal to that elicited by tasks using verbal/semantic stimuli (Fig. 1C) (Wendelken et al., 2008, 2011). Although these findings are seemingly contrary to the content-free role of the RLPFC proposed by Gilbert (2011), Wendelken et al. (2011) acknowledge a large amount of overlap between the dorsal and ventral regions. Thus, Gilbert's (2011) spotlight implementation of MVPA may not have been sensitive to content-specific storage with a high degree of overlap. Another possibility for the discrepancy in RLPFC content storage may be due to the multitasking in Gilbert's (2011) PM task, which entailed complex action rules. This additional complexity may have evoked abstract neural representations in rostral (multitasking) areas of the RLPFC, which could mask any content-specific organization in caudal (integration) RLPFC regions. In this scenario, the dorsal-ventral contentspecific gradation within the RLPFC is contingent upon rostrocaudal functional segregation, and suggests a more complex, nonlinear organization of the RLPFC. Indeed, recent research and an informal metaanalysis have indicated that the RLPFC may be considerably more compartmentalized 
than the organization posited in Figure 1 (Hampshire et al., 2011).

The complement of analyses implemented by Gilbert (2011), i.e., univariate, MVPA, and PPI, highlight the utility of these different techniques in characterizing the dynamic nature of the RLPFC. It was shown that bilateral RLPFC functionally couples with posterior content-storing regions to maintain delayed intentions. However, evidence suggests functional partitioning exists along the rostral-caudal axis, a content-specific distinction along the dorsal-ventral axis, and a lateralized specialization of the RLPFC. Thus, additional research is required to fully understand the functional architecture of the RLPFC and its role in prospective memory.

\section{References}

Adda CC, Castro LHM, Silva L, de Manreza MLG, Kashiara R (2008) Prospective memory and mesial temporal epilepsy associated with hippocampal sclerosis. Neuropsychologia 46:1954-1964.

Badre D, D'Esposito M (2009) Is the rostrocaudal axis of the frontal lobe hierarchical? Nat Rev Neurosci 10:659-669.

Bunge SA, Helskog EH, Wendelken C (2009) Left, but not right, rostrolateral prefrontal cortex meets a stringent test of the relational integration hypothesis. Neuroimage 46:338-342.

Burgess PW, Quayle A, Frith CD (2001) Brain regions involved in prospective memory as determined by positron emission tomography. Neuropsychologia 39:545-555.

Burgess PW, Dumontheil I, Gilbert SJ (2007) The gateway hypothesis of rostral prefrontal cortex (area 10) function. Trends Cogn Sci 11:290-298.

Fuster JM (2001) The prefrontal cortex - an update: time is of the essence. Neuron 30:319-333.
Gilbert SJ (2011) Decoding the content of delayed intentions. J Neurosci 31:2888-2894.

Hampshire A, Thompson R, Duncan J, Owen AM (2011) Lateral prefrontal cortex subregions make dissociable contributions during fluid reasoning. Cereb Cortex 21:1-10.

Koechlin E, Basso G, Pietrini P, Panzer S, Grafman J (1999) The role of the anterior prefrontal cortex in human cognition. Nature 399:148-151.

Wendelken C, Bunge SA (2010) Transitive inference: distinct contributions of rostrolateral prefrontal cortex and the hippocampus. J Cogn Neurosci 22:837-847.

Wendelken C, Nakhabenko D, Donohue SE, Carter CS, Bunge SA (2008) "Brain is to thought as stomach is to ?": investigating the role of rostrolateral prefrontal cortex in relational reasoning. J Cogn Neurosci 20:682-693.

Wendelken C, Chung D, Bunge SA (2011) Rostrolateral prefrontal cortex: domain-general or domain-sensitive? Hum Brain Mapp, in press. 